\title{
Angiography and the aetiology of heart failure
}

\author{
Carl IO Brookes, Paul Hart, Bruce E Keogh, John GF Cleland
}

\begin{abstract}
Summary
The diagnosis of idiopathic dilated cardiomyopathy should not be made without first performing a coronary angiogram. If the cause of heart failure is unknown this should be stated rather than attributing the cause to dilated cardiomyopathy. Severe ventricular dysfunction may improve dramatically after revascularisation in some cases of coronary disease. Preservation of $R$ waves on the surface electrocardiogram suggests the presence of hibernating myocardium but thallium scintigraphy or positron emission tomography scanning should be employed to investigate this further.
\end{abstract}

Keywords: coronary angiogram, heart failure

Recent studies have highlighted the inaccuracy of making a diagnosis of heart failure by clinical means alone. ${ }^{1,2}$ The aetiology of heart failure also has important therapeutic and prognostic implication $\mathrm{s}^{2,3}$ and cannot be ascertained without the help of additional investigations. ${ }^{4}$ The striking impact of angiotensin-converting enzyme (ACE) inhibitors on those patients with left ventricular systolic dysfunction and the potential benefit of surgery in others ${ }^{5,6}$ reinforce the need to pursue clarification of the underlying diagnosis.

We present the case of a young man with a globally hypokinetic left ventricle which demonstrates some of the pitfalls of relying on clinical evaluation alone.

\section{Case report}

A 48-year-old man, born in Pakistan but resident in the UK for over 20 years, presented with symptoms and signs of severe heart failure. He had recently been discharged from his local hospital with a diagnosis of dilated cardiomyopathy based on clinical and echocardiographic findings.

His current illness began four months prior to presentation with episodic shortness of breath and sputum production. He received multiple courses of antibiotics with no effect. Following this he travelled to India where he became increasingly short of breath with associated ankle swelling. He was treated by a local physician with a series of intramuscular injections of unknown composition but he continued to get worse. Direct questioning revealed no history of chest pain or palpitations. He did not smoke, abuse alcohol or illicit substances and there was no family history of ischaemic heart disease. He was known to have retinitis pigmentosa (as was his sister) but he was not ataxic or deaf. He had no other relevant past medical history. At the time of presentation he was taking captopril $12.5 \mathrm{mg}$ bid and frusemide $80 \mathrm{mg}$ od.

On examination he was short of breath on minimal exertion with a sinus tachycardia of 100 beats/min and a blood pressure of $100 /$ $60 \mathrm{mmHg}$. The venous pressure was elevated to the ear lobes with pulsatile hepatomegaly, ascites, and pitting oedema to the thighs. Auscultation revealed a third heart sound but no regurgitant murmurs. Fundoscopy confirmed retinitis pigmentosa but the rest of the neurological examination was unremarkable.

Initial investigations revealed a normal full blood count without an eosinophilia. The urea and electrolytes, thyroid function tests, plasma glucose, erythrocyte sedimentation rate and C-reactive protein were normal. His total cholesterol was $2.6 \mathrm{mmol} / \mathrm{l}$ with a normal profile. Radiologically he had cardiomegaly (CTR 19/29) with upper lobe venous distension. Electrocardiogram (ECG) showed sinus rhythm, with no pathological $Q$ waves and no evidence of left ventricular hypertrophy. Echocardiography demonstrated biventricular dilatation with global dysfunction (LVEDD $62 \mathrm{~mm}$ ) and mild mitral regurgitation (figure 1).

The normal inflammatory indices made an active myocarditis unlikely and there were no features to suggest ischaemic heart disease. A cardiomyopathy associated with retinitis pigmentosa, as occurs in the Kearns-Sayre syndrome, ${ }^{7}$ was possible but the lack of other neurological abnormalities virtually excluded this diagnosis. A diagnosis of idiopathic dilated cardiomyopathy seemed likely and he was treated with an increase in his medical therapy.

A serology screen for toxoplasmosis, coxsackie B and Borrelia was negative. Autoantibodies including anti-cardiolipin antibody, serum ferritin, serum vitamin $A$, serum phytanic acid levels and urine screening for homocystinuria were also normal. He exercised for four minutes of a modified Bruce protocol stopping because of shortness of breath with no chest pain or significant ECG changes.

Coronary angiography revealed severe triple vessel disease with global left ventricular hypokinesis (figure 2). The left ventricular end-diastolic pressure was $25 \mathrm{mmHg}$ and cardiac index $1.41 / \mathrm{min} / \mathrm{m}^{2}$. The ejection fraction on radionuclide ventriculography was $16^{\prime \prime}$.). Positron emission tomography (PET) sug- 


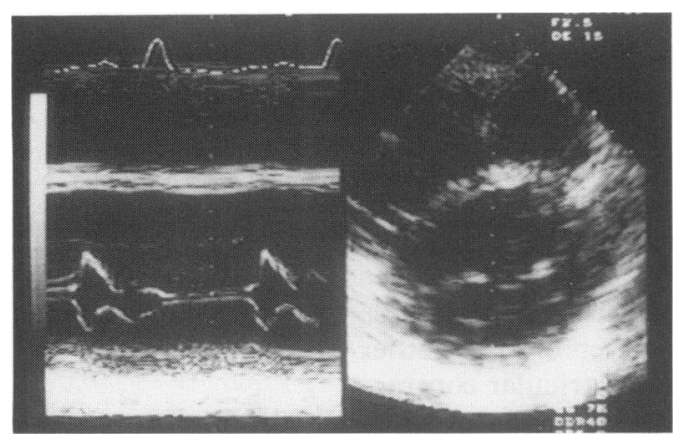

Figure 1 End-diastolic B-mode and M-mode echocardiographic frame demonstrating a dilated poorly contracting left ventricle.

gested widespread areas of non-contractile but viable myocardium.

Despite a dramatic diuresis with metolazone and frusemide and some symptomatic benefit on lisinopril $20 \mathrm{mg}$ od his improvement was not sustained. On the basis of his PET scan he was referred for coronary artery bypass surgery rather than cardiac transplantation. Although his postoperative course was stormy he was discharged home on the 17th postoperative day. Six months later his ejection fraction improved to $26 \%$ and at one year he is in NYHA class 1 , with an ejection fraction of $42 \%$, requiring no diuretic and being maintained on lisinopril alone.

\section{Discussion}

This report indicates the dangers of relying on clinical features alone to make the diagnosis of dilated cardiomyopathy.

The aetiology of dilated cardiomyopathy is poorly understood, ${ }^{8}$ although recent work suggests that $20 \%$ of cases may have a familial form of this disease which can partly be explained by a deletion in the promoter sequence of the gene that codes for cardiac dystrophin. ${ }^{9}$

However, the clinical diagnosis of dilated cardiomyopathy depends on the exclusion of other causes of ventricular dysfunction including specific myocardial diseases, valvular heart disease and important coronary artery disease.

Population-based studies show that the majority of patients with a presumptive diagnosis of dilated cardiomyopathy never undergo coronary angiography, making the potential for overestimating the prevalence of this condition large. In a review by Benton et $a l^{10}$ the authors highlight eight population-based studies of dilated cardiomyopathy, in five of which the frequency of angiography was less than $50 \%$ (range $34-48 \%$ ), the frequency of angiography not being stated in the other three. Given that both black race and low socioeconomic status are associated with a lower likelihood of angiography, this may explain, in part, the supposed increase of dilated cardiomyopathy in these groups. ${ }^{11-13}$

Furthermore, Figulla et $a l^{14}$ found that only $66 \%$ of patients referred for angiography with a presumptive diagnosis of dilated cardiomyopathy on the basis of non-invasive investigations were correctly diagnosed. Similarly, in
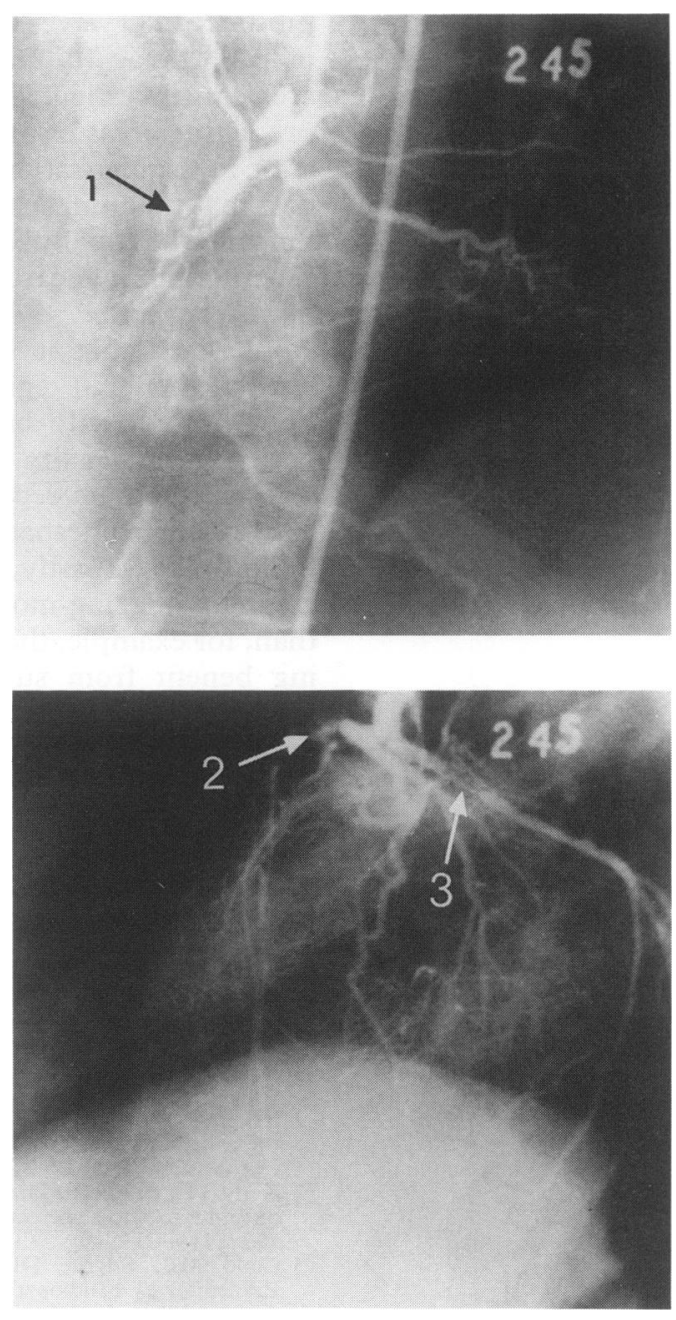

Figure 2 Coronary angiography showing an occluded right coronary artery (arrow 1), an occluded left anterior descending artery (arrow 2) and a tight proximal stenosis of the circumflex artery (arrow 3).

Benton's study ${ }^{10} 30.3 \%$ of patients with a discharge diagnosis of dilated cardiomyopathy had significant coronary artery disease as defined by more than $50 \%$ stenosis in one or more main coronary arteries.

These studies indicate that potentially treatable coronary artery disease will be missed in a large proportion of patients with a presumptive diagnosis of dilated cardiomyopathy if coronary angiography is not performed. However, coronary artery disease and dilated cardiomyopathy are not necessarily mutually exclusive and it remains a matter of individual judgement as to whether the severity of coronary artery disease is sufficient to account for the degree of left ventricular dysfunction in these cases.

The case above also highlights the potential of new imaging modalities to differentiate hibernating from infarcted myocardium. Although coronary angiography is the most accurate investigation for the detection of obstructive coronary artery lesions, it cannot distinguish between viable and non-viable myocardium. Similarly, while well preserved R waves on the surface ECG do increase the likelihood of finding viable myocardium, the 
presence of $Q$ waves do not indicate an absence of viable myocardium. ${ }^{15} \mathrm{~A}$ variety of techniques are being investigated which have some value in predicting areas of asynergy that will improve after revascularisation. ${ }^{16-18}$ Initial studies suggest that thallium scintigraphy and PET scanning may be equally sensitive in the detection of hibernating myocardium but PET may be more specific. . $^{190}$ However, it must be emphasized that it is not yet clear whether improvements in regional ventricular contraction translate into benefits in terms of morbidity and mortality.

The economic repercussions of identifying and treating hibernating myocardium need to be addressed. Firstly, it needs to be proven that complex imaging modalities are more reliable than, for example, the surface ECG in predicting benefit from surgery. Secondly, several small uncontrolled studies ${ }^{21-23}$ have indicated that revascularisation in patients with poor left ventricular function and severe coronary artery disease may improve prognosis which if confirmed, will lead to increasing surgical demand and longer waiting lists for bypass procedures. The plus side, however, is a potential reduction in the number of patients referred for cardiac transplantation.

1 Remes J, Miettinen H, Reunanen A, Pyorala K. Validity of the clinical diagnosis of heart failure in primary health care. the clinical diagnosis of heart fail

2 Kelly TL, Cremo R, Nielson C, Shabetai R. Prediction of outcome in late-stage cardiomyopathy. Am Heart $₹$ 1990; 119: $1111-21$

3 Codd MB, Sugrue DD, Gersh BJ, Melton LJ III. Epidemiology of idiopathic dilated and hypertrophic cardiomyopathy. A population based survey in Minnesota, 1975-1984. Circulation 1989; 80: 564-72.

4 Chakko S, Gheorghiade M. Estimating the severity of chronic heart failure: a clinical challenge for the 1990s. Am Heart $\mathcal{f} 1992 ; 124: 260-4$.

5 Elefteriades JA, Tolis G, Levi E, Mills LK, Zaret BL. Coronary artery bypass grafting in severe left ventricular Coronary artery bypass grafting in severe left ven.

6 Piggott JD, Kouchoukos NT, Oberman A, Cutter GR. Late 6 Piggott JD, Kouchoukos NT, Oberman A, Cutter GR. Late results of surgical and medical therapy in patients with
coronary artery disease and depressed left ventricular funccoronary artery disease and depressed left vent

7 Moraes CT, DiMauro S, Zeviani M et al. Mitochondrial DNA deletions in progressive external ophthalmoplegia and Kearns-Sayre syndrome. N Engl f Med 1989; 320: 1293-9.

8 Tamburro $P$, Wilber $D$. Sudden death in idiopathic dilated cardiomyopathy. Am Heart $\mathcal{F}$ 1992; 124: 1035-45.

9 William Dec G, Fuster V. Idiopathic dilated cardiomyopathy. $N$ Engl f Med 1994; 331: 1564-75.

10 Benton RE, Coughlin SS, Tefft MC. Predictors of coronary angiography in patients with IDCM: the Washington DC dilated cardiomyopathy study. $\mathcal{F}$ Clin Epidemiol 1994; 47: 501-11.

11 Hannan EL, Kilburn H, O'Donnell JF, et al. Interracial access to selected cardiac procedures for patients hospitalized with coronary artery disease in New York State. Med Care 1991; 29: 430-41.

12 Ford E, Cooper R, Castaner A, et al. Coronary arteriography and coronary bypass surgery among whites and other racial and coronary bypass surgery among whites and other racial
groups relative to hospital-based rates for CAD: findings from NHDS. Am $\mathcal{F}$ Public Health 1989; 79: 437-40.

\section{Learning point}

Potentially treatable coronary artery disease may be missed in patients with a presumptive diagnosis of dilated cardiomyopathy if coronary angiography is not performed

\section{Conclusion}

A confident diagnosis of dilated cardiomyopathy should not be made without coronary angiography. The lack of $Q$ waves on the surface ECG in patients with severe coronary artery disease and poor ventricular function may indicate the existence of underlying hibernating myocardium. If this is supported by either PET or thallium imaging, coronary artery bypass surgery should be considered The benefits of revascularisation may only become apparent after weeks or months and the mortality in the immediate postoperative period is likely to be high, especially in elderly patients with widespread atherosclerotic disease.

13 Coughlin SS, Labenberg JR, Tefft MC. Black-white differences in IDCM. Epidemiology 1993; 4: 165-72.

14 Figulla HR, Kellerman AB, Stille-Siegener M, et al. Significance of coronary angiograph, left heart catheterization and endomyocardial biopsy for the diagnosis of IDCM Am Heart F 1992; 124: 1251-7.

15 Banka VS, Bodenheimer MM, Helfant RH. Determinants of reversible asynergy - the native coronary circulation. Circulation 1975; 52: 810-6.

16 Dilzian V, Rocco TP, Freedman NM, Leon MB, Bonow RO. Enhanced detection of ischaemic but viable myocardium by the reinjection of thallium after stressredistributing imaging. $N$ Engl f Med 1990; 323: 141-6.

17 Iskandrian AS, Hakki A, Kane SA, et al. Rest and redistribution thallium myocardial scintigraphy to predict improvement in left ventricular function after coronary arter bypass grafting. Am $\mathcal{f}$ Cardiol 1983; 51: 1312-6.

18 Tillisch JH, Brunken R, Marshall R, et al. Reversibility of cardiac wall motion abnormalities predicted by PET. $N$ Engl $\Im$ Med 1986; 314: 884-8.

19 Bonow RO, Dilsizian V, Cuocolo A, Bacharach SL Identification of viable myocardium in patients with coronary artery disease and left ventricular dysfunction: comparison of thallium and PET imaging. Circulation 1991; 83 26-37.

20 Tamaki N, Ohtani H, Yamashita K, et al. Metabolic activity in the areas of new fill-in after thallium-201 reinjection comparison with PET using fluorine 18-deoxyglucose. $f$ Nucl Med 1991; 32: 673-8.

21 Dreyfus G, Duboc A, Blasco C, et al. Coronary surgery can be an alternative to heart transplantation in selected patien with end-stage ischaemic heart disease. Eur $\mathcal{F}$ Cardiothorac Surg 1993, 7: 482-8.

22 Carmelo A, Milano MD, William D, et al. Coronary artery bypass in patients wtih severely depressed ventricular function. Ann Thorac Surg 1993; 56: 487-93.

23 Magovern JA, Magovern GJ, Maher TD, et al. Operation for congestive heart failure: transplantation, bypass, and cardiomyoplasty. Ann Thorac Surg 1993; 56: 418-25. 